\title{
Urokinase, a promising candidate for fibrinolytic therapy for intracerebral hemorrhage
}

\author{
*Qiang Tan, MD,' Qianwei Chen, MD1 Yin Niu, MD,1 Zhou Feng, MD,' Lin Li, MD, ${ }^{1}$ Yihao Tao, MD, \\ Jun Tang, MD, ${ }^{1}$ Liming Yang, MD, ${ }^{1}$ Jing Guo, MD, ${ }^{2}$ Hua Feng, MD, PhD, ${ }^{1}$ Gang Zhu, MD, PhD, and \\ Zhi Chen, MD, PhD'
}

\begin{abstract}
${ }^{1}$ Department of Neurosurgery, Southwest Hospital, Third Military Medical University, Chongqing; and ${ }^{2}$ Department of Neurosurgery, 211st Hospital of PLA, Harbin, People's Republic of China
\end{abstract}

\begin{abstract}
OBJECTIVE Intracerebral hemorrhage $(\mathrm{ICH})$ is associated with a high rate of mortality and severe disability, while fibrinolysis for $\mathrm{ICH}$ evacuation is a possible treatment. However, reported adverse effects can counteract the benefits of fibrinolysis and limit the use of tissue-type plasminogen activator (tPA). Identifying appropriate fibrinolytics is still needed. Therefore, the authors here compared the use of urokinase-type plasminogen activator (UPA), an alternate thrombolytic, with that of IPA in a preclinical study.
\end{abstract}

METHODS Intracerebral hemorrhage was induced in adult male Sprague-Dawley rats by injecting autologous blood into the caudate, followed by intraclot fibrinolysis without drainage. Rats were randomized to receive uPA, tPA, or saline within the clot. Hematoma and perihematomal edema, brain water content, Evans blue fluorescence and neurological scores, matrix metalloproteinases (MMPs), MMP mRNA, blood-brain barrier (BBB) tight junction proteins, and nuclear factor-KB (NF-KB) activation were measured to evaluate the effects of these 2 drugs in $\mathrm{ICH}$.

RESULTS In comparison with tPA, uPA better ameliorated brain edema and promoted an improved outcome after ICH. In addition, uPA therapy more effectively upregulated BBB tight junction protein expression, which was partly attributed to the different effects of UPA and TPA on the regulation of MMPs and its related mRNA expression following ICH.

CONCLUSIONS This study provided evidence supporting the use of uPA for fibrinolytic therapy after ICH. Large animal experiments and clinical trials are required to further explore the efficacy and safety of uPA in ICH fibrinolysis.

http://thejns.org/doi/abs/10.3171/2016.1.JNS152287

KEY WORDS intracerebral hemorrhage; urokinase-type plasminogen activator; tissue-type plasminogen activator; fibrinolytic therapy; perihematomal edema; vascular disorders

$\mathrm{S}$ PONTANEOUS intracerebral hemorrhage (ICH) is the most devastating type of stroke worldwide, and currently there are few treatment strategies for $\mathrm{ICH}$ induced brain injury. ${ }^{20}$ The physical effects of hematoma (mass effect) as well as perihematomal edema (PHE) can worsen patient outcomes after ICH..$^{6,7,36}$

In recent years, stereotactic aspiration and subsequent fibrinolysis have been developed and, compared with the conventional surgical removal of ICH, have been considered a less invasive and more effective treatment modality for spontaneous ICH given their limited damage to peripheral normal brain tissues..$^{25,26,39}$ So far, tissue-type plasminogen activator (tPA) is the most widely used agent for
ICH fibrinolysis. ${ }^{39}$ However, beyond its beneficial effect of resolving hematoma, many other side effects, such as the proedema, proinflammatory, and proneurotoxic effects, have been observed in preclinical trials..$^{12,14,21,29,33-35}$ Thus, the use of one or the other types of fibrinolytics remains empirical. In recent years, urokinase-type plasminogen activator (uPA), an older thrombolytic drug (not available in the United States since 1999), has gradually resurfaced as a possible fibrinolytic treatment. ${ }^{13,14} \mathrm{~A}$ growing number of clinical research studies have reported that uPA not only increases the rate of clot resolution but also improves patient outcome in ICH fibrinolysis. . $, 8,23,38$ Nevertheless, no preclinical or clinical studies have evaluated the differen-

ABBREVIATIONS BBB = blood-brain barrier; EB = Evans blue; GAPDH = glyceraldehyde 3-phosphate dehydrogenase; ICH = intracerebral hemorrhage; IVH = intraventricular hemorrhage; MISTIE = minimally invasive surgery plus rtPA for ICH evacuation; MMP = matrix metalloproteinase; NF-kB = nuclear factor-kB; $\mathrm{PHE}=$ perihematomal edema; $\mathrm{RBC}$ = red blood cell; RT-PCR = real-time polymerase chain reaction; tPA = tissue-type plasminogen activator; $\mathrm{uPA}=$ urokinase-type plasminogen activator; $v W F=$ von Willebrand factor.

SUBMITTED September 29, 2015. ACCEPTED January 29, 2016.

INCLUDE WHEN CITING Published online April 22, 2016; DOI: 10.3171/2016.1.JNS152287.

* Drs. Tan and Q. Chen contributed equally to this work 
tial effect of these 2 agents for fibrinolytic therapy following ICH. We hypothesized that uPA could provide greater benefits than TPA when used for ICH fibrinolysis. Thus, in the present study, we decided to compare the efficacy of these 2 fibrinolytics in an ICH model in rats.

\section{Methods Animals}

One hundred thirty adult male Sprague-Dawley rats weighing 250-350 g were used in this study (Third Military Medical University). Animals were housed under controlled, pathogen-free conditions with free access to pellet food and water. Surgical procedures were performed aseptically, and standard microsurgical techniques were used with an operating microscope. At the study end point, while under deep anesthesia, animals were killed using an overdose of intraperitoneal pentobarbital. All efforts were made to minimize suffering and animal numbers, according to the Guide for the Care and Use of Laboratory Animals, and the study protocol was approved by the Animal Care and Use Committee at the Third Military Medical University.

\section{Surgical Procedures}

To induce ICH, animals were anesthetized with pentobarbital (40 mg/kg intraperitoneal), and the right femoral artery was catheterized to monitor arterial blood pressure, blood $\mathrm{pH}, \mathrm{PaO}_{2}, \mathrm{PaCO}_{2}$, and glucose levels 1 hour before and 6 hours after ICH (Table 1). A feedback-controlled heating pad was used to maintain body temperature at $37^{\circ} \mathrm{C}$. A cranial bur hole $(1 \mathrm{~mm})$ was drilled, and a 29-gauge needle was inserted stereotactically into the right caudate nucleus (coordinates: $0.2 \mathrm{~mm}$ anterior, 5.5 $\mathrm{mm}$ ventral, and $3.5 \mathrm{~mm}$ lateral to the bregma). In total, $100 \mu \mathrm{l}$ of autologous arterial blood was infused at a rate of $10 \mu \mathrm{l} /$ minute by using a microinfusion pump in each rat. The sham groups required only a needle injection into the right caudate nucleus.

\section{Experimental Groups and Fibrinolytic Therapy}

There were 2 parts to this study. First, 70 rats were randomly divided into 5 groups of 14 rats each: sham, $\mathrm{ICH}, \mathrm{ICH}+$ saline, ICH+tPA, and ICH+uPA. Thirty minutes after hematoma placement, $2 \mu \mathrm{l}$ of saline, $2 \mu \mathrm{l}$ of tPA (10 $\mu \mathrm{g} / \mu \mathrm{l}$, Actilyse, Boehringer Ingelheim International), or $2 \mu \mathrm{l}$ of uPA (100 IU/ $\mu \mathrm{l}$, urokinase for injection, No. H44020647, approved by state, Livzon Pharmaceutical Group Co.) was injected through an intracerebral catheter with a microinfusion pump, into the core of the hematoma. In the present study, the dose regimen of tPA $(20 \mu \mathrm{g})$ was used, as described in a previous study ${ }^{28}$ of a Wistar rat model of ICH in which Wistar rats received a 50- $\mu$ l blood injection and a subsequent $12-\mu \mathrm{g}$ recombinant tPA (rtPA) treatment. For uPA, no dose study in rat ICH fibrinolysis has been reported as yet. In a preclinical study of intraventricular hemorrhage (IVH) fibrinolysis, Gaberel et al. reviewed the relevant literature and proposed that 10,000 IU of uPA is equivalent to $1 \mathrm{mg}$ of tPA..$^{14}$ Thus, $200 \mathrm{IU}$ of uPA was selected as an amount comparable to $20 \mu \mathrm{g}$ of tPA in our study. Furthermore, according to the proportion between hematoma size and drug dosage, the established
TABLE 1. Physiological parameters of rat 1 hour before and 6 hours after ICH

\begin{tabular}{lccc}
\hline \multicolumn{1}{c}{ Variable } & Before ICH & $\mathrm{ICH}+\mathrm{tPA}$ & $\mathrm{ICH}+\mathrm{uPA}$ \\
\hline No. of rats & 4 & 4 & 4 \\
\hline $\mathrm{MABP}(\mathrm{mm} \mathrm{Hg})$ & $105.6 \pm 7.3$ & $109.4 \pm 9.8$ & $110.2 \pm 11.5$ \\
\hline $\mathrm{pH}$ & $7.12 \pm 0.07$ & $7.24 \pm 0.09$ & $7.21 \pm 0.08$ \\
\hline $\mathrm{PaO}_{2}(\mathrm{~mm} \mathrm{Hg})$ & $114.6 \pm 9.4$ & $108.5 \pm 10.7$ & $113.1 \pm 12.4$ \\
\hline $\mathrm{PaCO}_{2}(\mathrm{~mm} \mathrm{Hg})$ & $45.2 \pm 5.1$ & $46.3 \pm 3.2$ & $47.5 \pm 4.8$ \\
\hline Glucose $(\mathrm{mg} / \mathrm{dl})$ & $197.1 \pm 15.4$ & $212.3 \pm 15.9$ & $217.3 \pm 17.5$ \\
\hline
\end{tabular}

$\mathrm{MABP}=$ mean arterial blood pressure.

dose of tPA and UPA are equivalent to the dosage in ICH patients. ${ }^{8,23,26}$ To avoid additional injury to the normal perihematomal brain tissue during intraclot drainage, we used fibrinolytic injection alone rather than fibrinolytic injection with intraclot drainage. The bur hole was sealed with bone wax, and the skin incision was closed with sutures after the needle was removed. Animals were allowed to recover in the conditions described above until the testing phase. All rats were killed on Day 3 after ICH for brain water content (8 rats/group) and Evans blue (EB) fluorescence (6 rats/ group) after performing MRI and behavioral tests.

Second, 54 rats were randomly divided into 3 groups of 18 rats each: ICH+saline, ICH+tPA, or ICH+uPA. After fibrinolysis, as described above, all rats were killed on Day 3 after ICH for Western blot (9 rats/group: 4 for matrix metalloproteinases [MMPs] and tight junction protein $\mathrm{ZO}-1$ and 5 for nuclear factor $[\mathrm{NF}]-\mathrm{\kappa B})$, real-time polymerase chain reaction (RT-PCR; 4 rats/group) assay, and immunofluorescence (5 rats/group). A total of 6 rats died within 24 hours of the injection of autologous arterial blood.

\section{Magnetic Resonance Imaging and Volume Measurement}

Rats were anesthetized with a $2 \%$ isoflurane/air mixture throughout the MRI examination performed on Day 3 after ICH. Magnetic resonance imaging was performed in a 7.0-T Varian MR scanner (Bruker Corp.) with a T2 fast spin-echo sequence using an FOV $35 \times 35 \mathrm{~mm}$ and 17 coronal slices (1.0-mm thickness). Volumes were calculated as previously described. ${ }^{6,10}$ Hematoma and PHE were outlined, and the areas were measured. Volumes were assessed by calculating the areas of all slices and multiplying by the section thickness. All image analyses were performed by 2 observers blinded to the rat treatment factors by using ImageJ (National Institutes of Health).

\section{Brain Water Content}

Three days after ICH and MRI, rats were killed. Brains were removed and divided into 2 hemispheres and the cerebellum, as previously described. ${ }^{11}$ The wet weights were determined immediately. Then, the brain sections were dried at $100^{\circ} \mathrm{C}$ for 24 hours before obtaining the dry weights. The percentage of water content was calculated as follows: (wet weight - dry weight)/wet weight $\times 100 \%$. The percentage of water content was calculated by $2 \mathrm{ob}-$ servers blinded to the rat treatment factors.

\section{Evans Blue Fluorescence}

Vascular permeability was determined by measuring 
the fluorescence intensity of EB dye (Aladdin US) extravasation 3 days after blood infusion. Rats were injected with $2 \%$ EB dye $(5 \mathrm{ml} / \mathrm{kg})$ via the tail vein 60 minutes before perfusion and brain removal, as previously described. ${ }^{6}$ Then, the brains were removed in preparation for coronal brain sections. Red autofluorescence of EB was observed on the slides, as previously described.9,22 The mean red autofluorescence of EB was evaluated by 2 observers blinded to rat treatment factors.

\section{Corner Turn Test}

The corner turn test was performed 3 days after ICH and before MRI, as described previously. ${ }^{18}$ Briefly, the rat was allowed to proceed into a corner, whose angle was $30^{\circ}$. To exit the corner, the rat could turn to either the left or the right, and that direction was recorded. The test was repeated 10-15 times, with at least 30 seconds between trials, and the percentage of right turns was calculated. The mean neurological score was evaluated by 2 blinded observers.

\section{Forelimb Placing Test}

The forelimb-placing test was performed 3 days after ICH and before MRI, as described previously. ${ }^{18}$ Briefly, each rat was tested 10 times for each forelimb, and the percentage of trials in which the rat placed the appropriate forelimb on the edge of the countertop in response to vibrissae stimulation was determined. Testers were highly experienced and blinded to the condition of the animal. The mean neurological score was evaluated by 2 blinded observers.

\section{Real-Time PCR}

The PCR was performed and analyzed, as previously described ${ }^{30}$ For RT-PCR MMP gene expression analysis, rats (4/group) were sacrificed by decapitation 3 days after ICH. The brains were then dissected $2 \mathrm{~mm}$ anteriorly and $2 \mathrm{~mm}$ posteriorly to the needle entry site (easily identifiable on the brain surface) and were divided into separate hemispheres along the midline. Next, the striatum on each side of the brain was separated from the surrounding white matter and cortex and was used for RNA extraction. In the ICH animals, this area included most of the hematoma and a surrounding region of striatal tissue. Primers were designed with the Primer3 Output program (http://primer3.ut.ee; Table 2). Total RNA was extracted using TRIzol reagent (Invitrogen). A positive standard curve for each primer was obtained using a serially diluted cDNA (complementary DNA) sample mixture. Gene expression was quantified with standard samples and normalized with glyceraldehyde 3-phosphate dehydrogenase (GAPDH). The data are expressed as normalized mRNA expression (fold mRNA [messenger RNA] increase). All data analyses were performed in a blinded manner by 2 observers.

\section{Western Blot Analysis}

Western blot analysis was performed, as previously described. ${ }^{16,31}$ The brains were perfused with saline before decapitation on Day 3 after injection. The perihematomal brain tissue (4-mm-thick brain tissue around the hema-
TABLE 2. Primers used for RT-PCR

\begin{tabular}{cc}
\hline $\begin{array}{c}\text { Gene \& GenBank } \\
\text { Accession No. }\end{array}$ & \multicolumn{1}{c}{ Primer Sequences } \\
\hline MMP-2 & \\
\hline NM031054 & AGAAGGCTGTGTCTTCGCA (FP) \\
\hline MMP-9 & AAAGGCAGCGTCTACTTGCT (RP) \\
\hline NM031055 & CTGCCTGCACCACTAAAGG (FP) \\
\hline MMP-12 & GAAGACGAAGGGGAAGACG (RP) \\
\hline NM053963 & CTGGGCAACTGGACACCT (FP) \\
\hline & CTACATCCGCACGCTTCA (RP) \\
\hline
\end{tabular}

$\mathrm{FP}=$ forward primer; $\mathrm{RP}=$ reverse primer.

toma) was sampled. The primary antibodies included anti-MMP-2 (1:1000, CST), anti-MMP-12 (1:1000, Abcam), anti-ZO-1 (1:500, Santa Cruz), anti-NF-кB p65 (1:1000, CST), anti-phospho-NF-кB p65 (1:1000, CST), anti-GAPDH (1:1000, Santa Cruz), anti- $\beta$-actin (1:1000, Beyotime). The relative densities of the bands were analyzed using NIH ImageJ software. All data analyses were performed in a blinded manner by 2 observers.

\section{Immunofluorescence}

Immunofluorescence staining of brain tissue was performed on fixed frozen sections, as previously described. ${ }^{6}$ Rats were anesthetized with pentobarbital $(100 \mathrm{mg} / \mathrm{kg}$ intraperitoneal) and perfused with 4\% paraformaldehyde in $0.1 \mathrm{~mol} / \mathrm{L}$ phosphate-buffered saline $(\mathrm{pH}$ 7.4) on Day 3 after ICH. The brains were removed and kept in $4 \%$ paraformaldehyde for 4-6 hours and then immersed in 30\% sucrose for 3-4 days at $4^{\circ} \mathrm{C}$. The brains were embedded in an optimal cutting temperature compound (Sakura Finetek USA), and 18 -mm-thick slices were cut using a cryostat. The following primary antibodies were used: goat antiZO-1 (1:200, Santa Cruz), mouse anti-von Willebrand factor (vWF; 1:200, Santa Cruz), and mouse anti-claudin-5 (1:200, Invitrogen). Appropriate secondary antibodies were incubated with the specimens for 3 hours at $37^{\circ} \mathrm{C}$. Immunofluorescence was examined using a confocal fluorescence microscope (LSM780, Zeiss).

\section{Statistical Analysis}

Sample size estimations were made by power analysis using a Type I error rate of 0.05 and a power of 0.8 on a 2 -sided test. Results are expressed as the means \pm standard deviation. For each type of experiment, data were obtained from at least 3 independent measurements. Statistical analysis of the results was conducted using a 1-way ANOVA, followed by the least significant difference test or Newman-Keuls test. Differences were considered significant at $\mathrm{p}<0.05$. All data were strictly analyzed in a blinded manner by 2 observers.

\section{Results}

\section{Effect of tPA and UPA on Hematoma Resolution and Brain Edema Following ICH}

Three days after ICH, although both IPA and uPA treat- 
ment reduced the volumes of PHE ( $\mathrm{p}<0.01$ vs vehicle; Fig. 1A and B) and hematoma ( $p<0.05$ vs vehicle; Fig. $1 \mathrm{C})$, uPA was more effective in reducing PHE (7.42 \pm 3.31 vs $12.15 \pm 4.64 \mathrm{~mm}^{3}, \mathrm{p}<0.05$ vs tPA). In addition, significantly decreased brain water content in the ipsilateral hemisphere was observed in the uPA group (79.84 \pm 0.41 vs $80.89 \pm 0.68 \mathrm{~mm}^{3}, \mathrm{p}<0.05$ vs vehicle; Fig. 1D) rather than the tPA group ( $p>0.05$ vs vehicle).

\section{Effect of tPA and uPA on Blood-Brain Barrier Protection and Neurological Recovery After ICH}

In comparison with tPA, uPA therapy more significantly attenuated blood-brain barrier (BBB) injury and promoted functional recovery on Day 3 following ICH. Quantification of perivascular EB fluorescence revealed that uPA, in comparison with tPA, more effectively prevented $\mathrm{BBB}$ destruction and decreased $\mathrm{EB}$ extravasation from the vasculature (uPA $69.34 \pm 22.62 \mathrm{~mm}^{3}$ vs tPA 87.31 $\pm 26.78 \mathrm{~mm}^{3}, \mathrm{p}<0.05$; Fig. $2 \mathrm{~A}$ and B). During the behavior tests on Day 3 post-ICH thrombolytic therapy, the tPA group had a trend toward a higher neurological score than did the vehicle group, but there was no significant difference (tPA vs vehicle, $\mathrm{p}>0.05$; Fig. $2 \mathrm{C}$ and D); the uPA group had a lower corner turn score (uPA 69.82 \pm 23.95 vs vehicle $90.49 \pm 21.54, \mathrm{p}<0.05)$ and higher forelimb placing score (uPA $49.24 \pm 28.17$ vs vehicle $17.13 \pm 13.80, \mathrm{p}<$ $0.05)$ than did the control groups.

\section{Effect of tPA and UPA on MMP and BBB Tight Junction Protein Level Regulation After ICH}

The uPA therapy more effectively upregulated BBB tight junction protein expression than did tPA, which was partly attributed to the different effects of uPA and tPA on the regulation of MMPs and related mRNA expression after ICH. As shown in Fig. 3A, both uPA and tPA effectively decreased the MMP-12 mRNA expression ( $\mathrm{p}<$ 0.05 vs vehicle) on Day 3 after ICH. However, only uPA significantly reduced the MMP-2 mRNA expression level (uPA $0.87 \pm 0.05$ vs vehicle $1.03 \pm 0.11, \mathrm{p}<0.05$ ). In addition, more remarkable MMP-9 mRNA expression was observed following treatment with tPA in comparison with uPA (tPA $1.03 \pm 0.23$ vs $0.87 \pm 0.29$ uPA, $\mathrm{p}<0.05$ ). Moreover, the corresponding Western blotting assays revealed trends in MMP expression levels similar to those in the mRNA assays (Fig. 3B). Despite the increased MMP-9 expression after ICH thrombolysis, both uPA and tPA effectively protected $\mathrm{ZO}-1$ ( $\mathrm{p}<0.05$ and 0.01 , respectively, vs vehicle; Fig. 4A and B) and claudin-5 ( $\mathrm{p}<0.05$ and 0.01 , respectively, vs vehicle; Fig. 4C-E) from degeneration. In particular, uPA had a more prominent effect $(\mathrm{p}<$ 0.05 , uPA vs tPA) in these processes.

\section{Effect of tPA and uPA on NF-kB Pathway Activation After ICH}

To study the role of tPA and uPA on NF-кB pathway

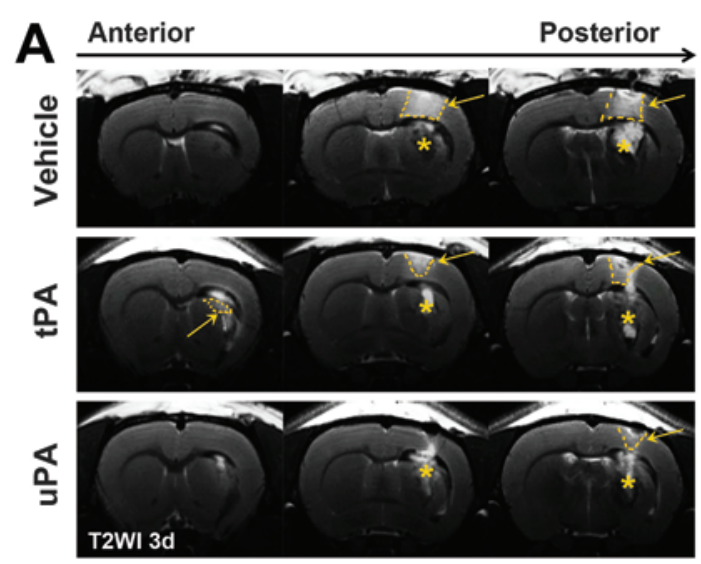

B


FIG. 1. Effect of tPA and UPA on hematoma evacuation and brain edema following ICH. A: Representative T2-weighted images obtained on Day 3 after ICH. Arrows denote the PHE area (high signal zone), and asterisks denote the ICH. Quantification of volumes of PHE (B) and hematoma (C) according to the related T2-weighted images (14 rats/group). Brain water content determination (D) in ipsilateral hemisphere 3 days after ICH (8 rats/group). Results are presented as the means \pm standard deviation. * $p<$ $0.05,{ }^{* *} p<0.01$. NS = no significance; T2WI $3 d=$ T2-weighted imaging Day 3 after ICH. Figure is available in color online only. 

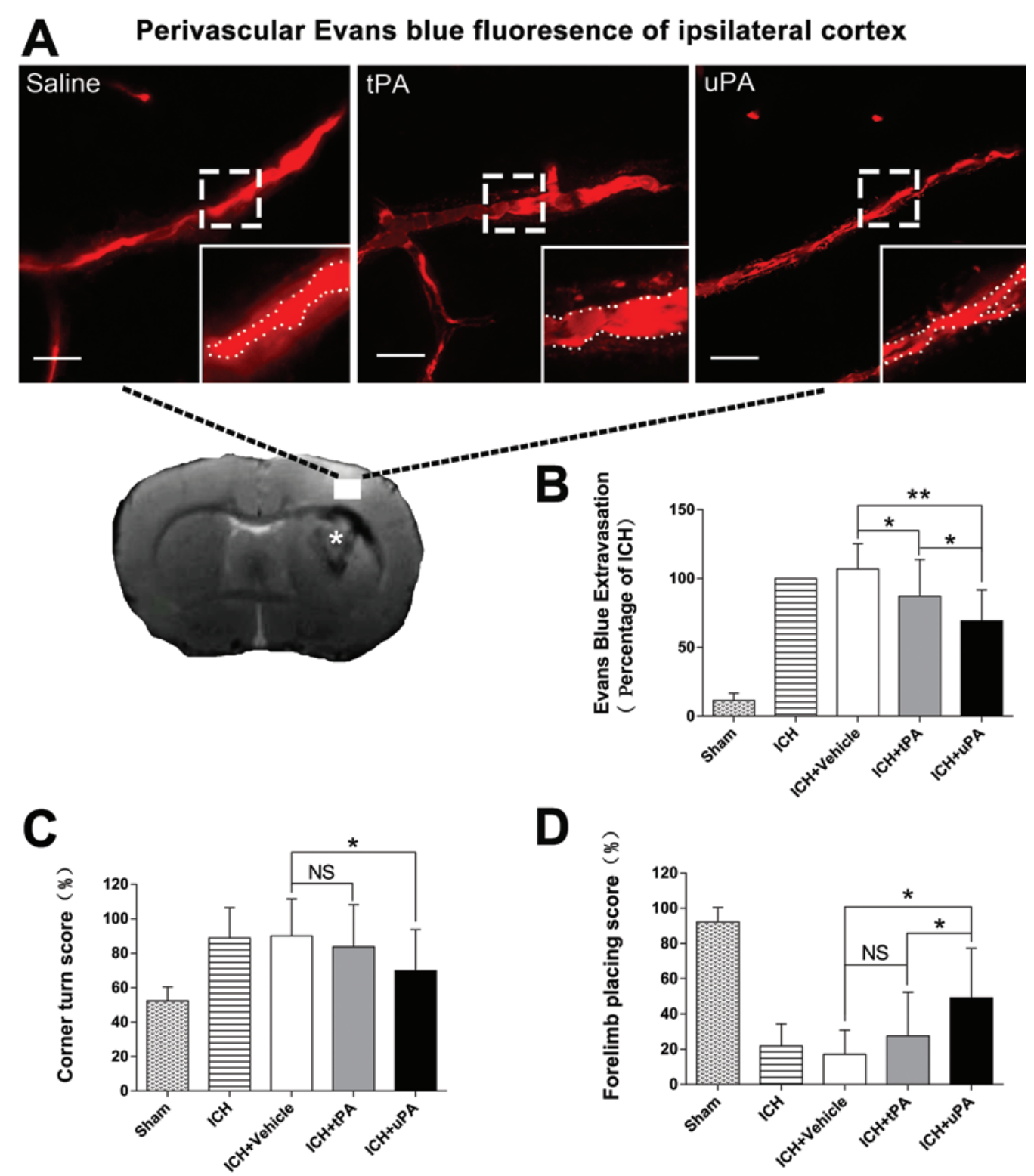

FIG. 2. Effect of tPA and UPA on BBB protection and neurological recovery after ICH. A: Representative confocal images display the perivascular EB dye leakage from vessels of the ipsilateral cortex. Each dashed white square represents the respective magnified inset. Asterisk in the brain section denotes the intracerebral hematoma; the solid white square in the brain section denotes the PHE. B: Quantification of perivascular EB fluorescence on Day 3 after ICH (6 rats/group). C: Results of corner turn test on Day 3 after post-ICH thrombolytic therapy (14 rats/group). D: Results of forelimb placing test on Day 3 after post-ICH thrombolytic therapy. Results presented as the means \pm standard deviation. ${ }^{*} p<0.05,{ }^{* *} p<0.01$. Figure is available in color online only.

activation after ICH, we performed Western blot analysis for phospho-p65 and total p65 (Fig. 5A). Although there was no significant difference among vehicle, tPA therapy, and uPA therapy on the expression of p65 (vehicle 1.290 $\pm 0.4106, \mathrm{uPA} 1.264 \pm 0.4662$, tPA $1.325 \pm 0.2809, \mathrm{p}>$ 0.05 ; Fig. 5B), we observed a significant exacerbation of phospho-p65 expression following treatment with tPA rather than with uPA or vehicle (tPA $0.7251 \pm 0.2109$ vs uPA $0.3991 \pm 0.1332, p<0.05 ;$ tPA vs vehicle $0.2429 \pm$ $0.08328, p<0.01)$. As phosphorylation of $\mathrm{p} 65$ has been seen as a key factor of the canonical NF- $\kappa \mathrm{B}$ pathway, ${ }_{15,17}$ we normalized phospho-p65 to p-65 and quantified the
$\mathrm{NF}-\kappa \mathrm{B}$ pathway activation by the relative densities of each band (tPA $0.5474 \pm 0.1592$ vs uPA $0.3158 \pm 0.1054, \mathrm{p}<$ 0.05 ; tPA vs vehicle $0.1884 \pm 0.06457, \mathrm{p}<0.01$; Fig. $5 \mathrm{C}$ ). Together, our data demonstrated that tPA resulted in a significant exacerbation of NF- $\mathrm{KB}$ pathway activation for ICH fibrinolysis.

\section{Discussion}

We used a rat model of $\mathrm{ICH}$, followed by intracerebral hematoma fibrinolysis with either uPA or tPA. Although both uPA and tPA displayed similar efficacy in reducing 
A

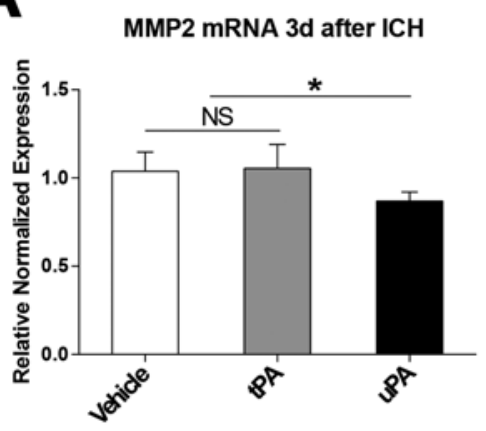

B
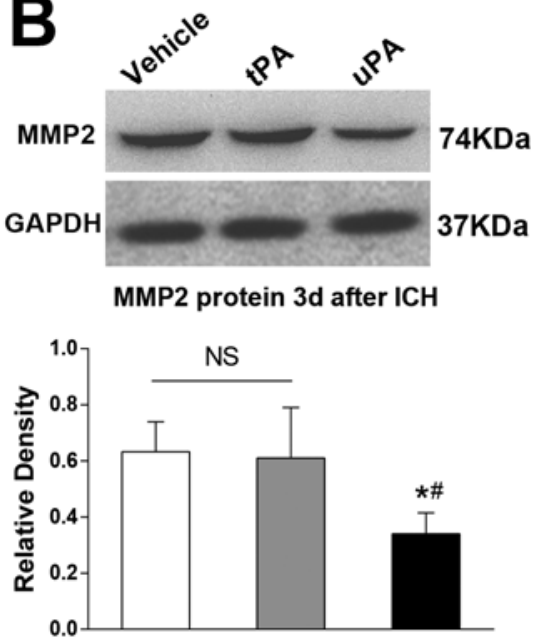
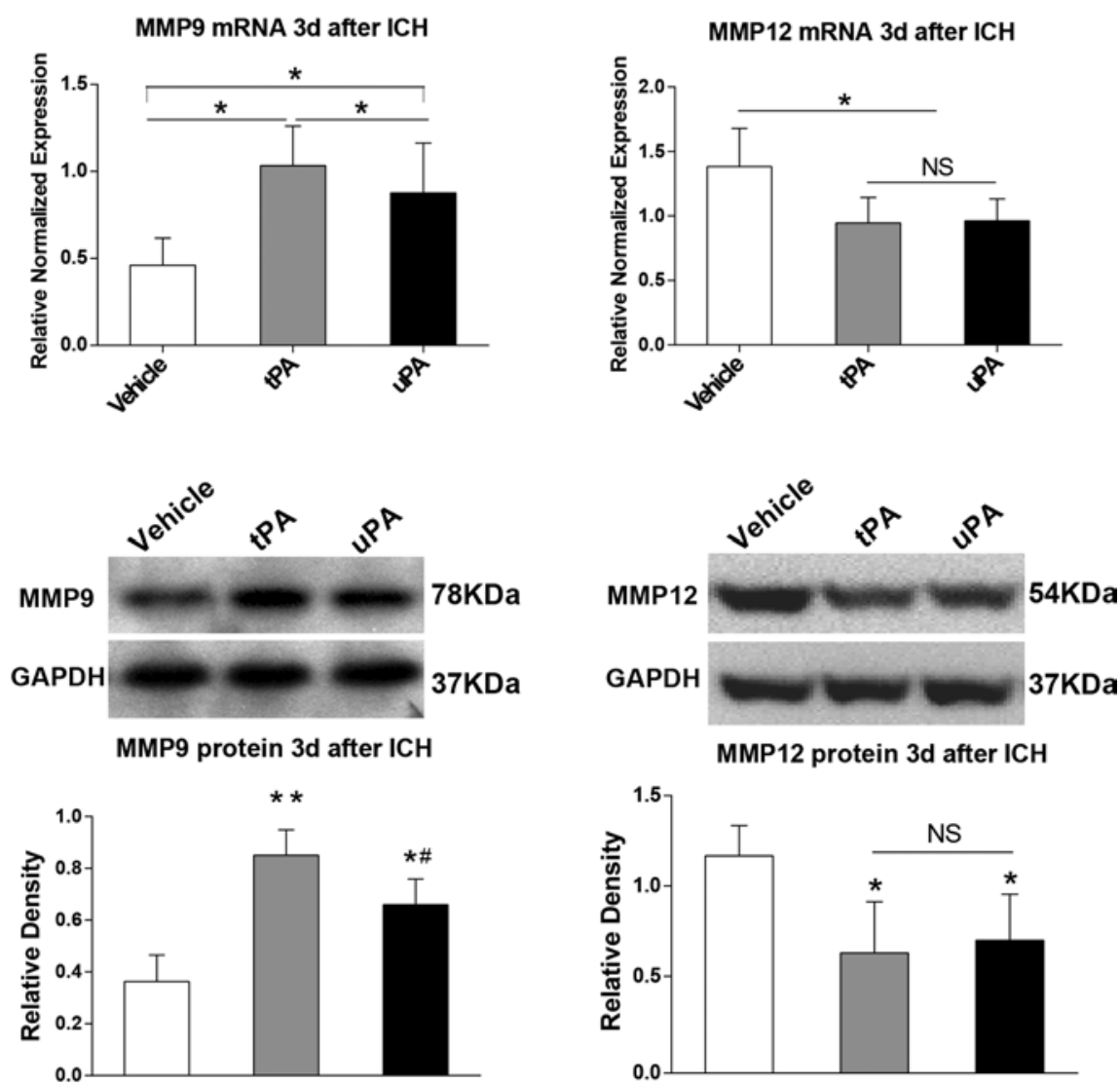

FIG. 3. Effect of tPA and UPA on MMP and MMP mRNA levels regulation after ICH. A: Relative normalized expression levels of MMP mRNAs in the perihematomal area on Day 3 (3d) after ICH (4 rats/group). B: Representative Western blot analysis and quantification of the relative density of the band of MMPs (4 rats/group). Results are presented as the means \pm standard deviation. ${ }^{*} p<0.05,{ }^{* *} p<0.01$ vs vehicle group, $\# p<0.05$ vs the tPA group.

the hematoma volume, uPA-mediated fibrinolysis alleviated PHE and promoted functional recovery better than did tPA in ICH rats.

Although hematoma evacuation can effectively reduce PHE, ${ }^{26}$ the surgical evacuation of deep hemorrhages is not always feasible, as sometimes it can require traversing a significant area of normal cerebral tissue. Thus, minimally invasive methods are essential. Minimally invasive surgery plus thrombolysis for ICH represents a minimally traumatic method of evacuating both superficial and deep hemorrhages. Over the last decade, several groups have reported significant results favoring accelerated clot thrombolysis with an acceptable safety profile., ${ }^{4,24,42}$ Recently, the minimally invasive surgery plus rtPA for ICH evacuation (MISTIE) Phase II trial tested the safety and efficacy of hematoma evacuation after ICH and demonstrated a significant reduction in hematoma volume as well as in PHE. ${ }^{26}$ In agreement with results in the aforementioned clinical trials, we also observed a significant reduction in hematoma and PHE in rat models of ICH after thrombolytic therapy in the present study. Moreover, we exposed these thrombolysis-treated animals to behavioral tests and found a remarkable recovery of motor function, which is, to our knowledge, the first time the behavioral tests have been conducted. At present, the MISTIE Phase III trial is under way to evaluate whether the reduction in hematoma size and edema translates to a reduced mortality and improved functional outcome.

During thrombolytic therapy in ICH rats in our study, without any attempts to aspirate the clot or the lysed material, as has been described elsewhere, ${ }^{27}$ the fibrinolytics injection successfully accelerated intracerebral hematoma resolution. Although relatively little is known about the mechanisms involved in hematoma resolution and how they are regulated, phagocytosis of red blood cells (RBCs) by microglia and/or infiltrating macrophages has also been suggested to have a role in resolution. ${ }^{40,41}$ In this study, thrombolytics penetrated the clot and degraded the fibrin, which may produce more room for interactions between microglia and/or infiltrating macrophages and free RBCs. Thus, the greater RBC phagocytosis by macrophages in the thrombolytic group, in comparison with the control group, may contribute to accelerated clot evacuation. Future studies will need to delineate the mechanisms involved in this phenomenon. Because enhancing the endogenous mechanisms related to hematoma resolution is an alternative strategy in ICH treatment, it can be used to overcome the brain trauma associated with surgical clot removal. ${ }^{39}$ The blood injection model used in the present study may not be the best model to assess the effects of 

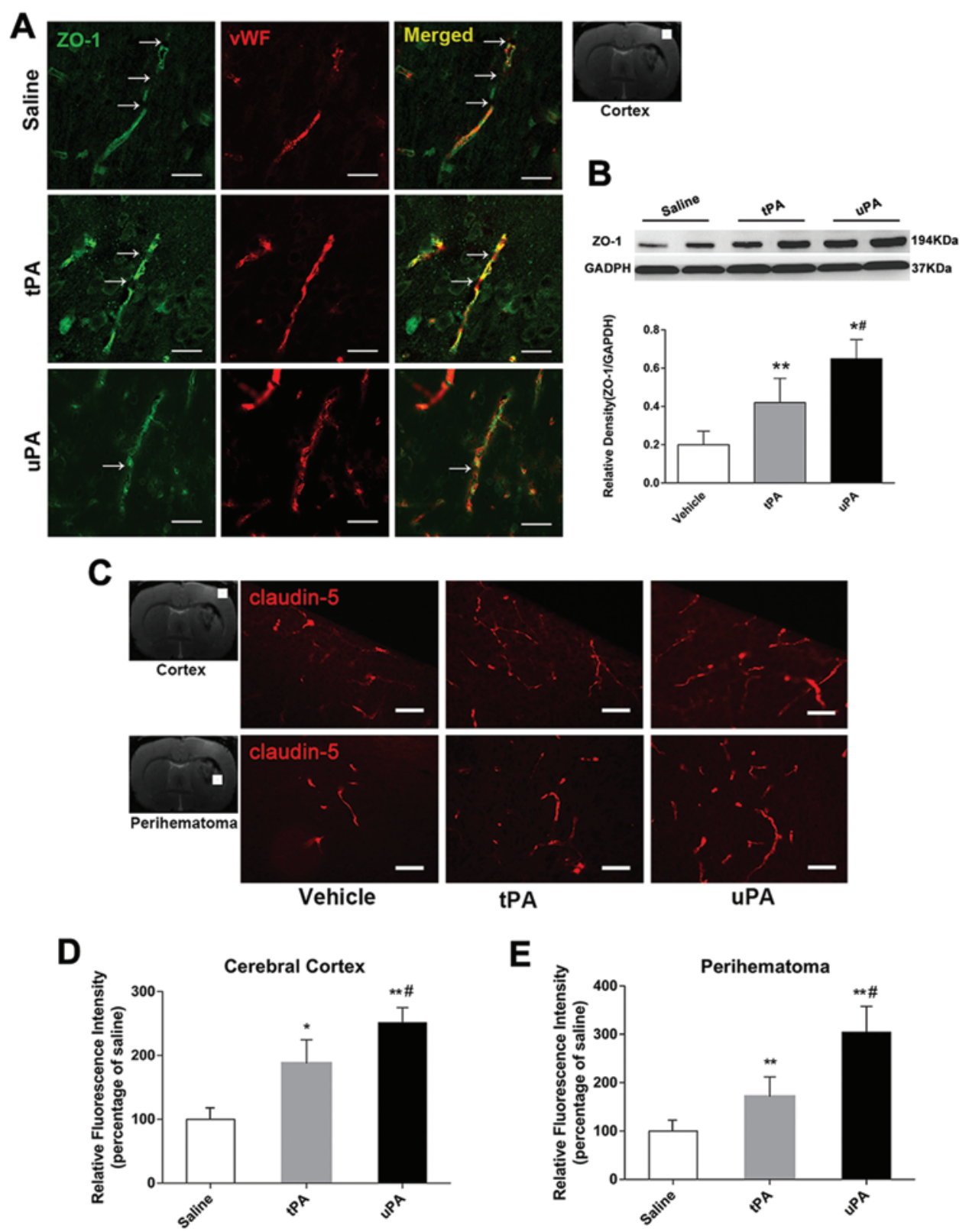

FIG. 4. Effect of tPA and UPA on BBB tight junction protein expression regulation after ICH. A: Representative photographs of immunofluorescence staining (arrows) for BBB tight junction protein (ZO-1, green) expression in endothelia (vWF, red) in the perihematomal area 3 days following ICH (5 rats/group). Western blot assay (B) for the ZO-1 level in the perihematomal area on Day 3 after ICH (4 rats/group). Immunofluorescence staining for claudin-5 (C) and relative fluorescence intensity assay to control group in the ipsilateral cortex (D) and perihematomal region (E, 5 rats/group). ${ }^{*} p<0.05,{ }^{* *} p<0.01$ vs the vehicle group, \#p $<0.05$ vs the tPA group. Bar $=10 \mu \mathrm{m}(\mathrm{A}), 20 \mu \mathrm{m}(\mathrm{C})$. Figure is available in color online only.

fibrinolytics (especially without aspiration) on hematoma volume reduction, and other types of models are required in the future.

As noted above, many researchers have reported reduced PHE after tPA thrombolysis and aspiration either in large animal models or in humans with $\mathrm{ICH} .{ }^{26,28,32,37,42}$ However, it should be noted that the adverse effects of tPA are still under debate. Preclinical studies have reported that tPA can have neurotoxic effects by inducing inflammation, disrupting the $\mathrm{BBB}$, causing neuronal degeneration, and exacerbating cerebral edema..$^{12,14,21,29,34,35} \mathrm{~A}$ recent meta-analysis conducted by Gaberel et al. has suggested that UPA, an alternate thrombolytic, is superior to tPA for IVH clot evacuation. ${ }^{13}$ Subsequently, Gaberel et al. designed a preclinical experiment to evaluate the differential impact of uPA versus tPA in a rat model of IVH and found that only uPA significantly improved functional recovery. ${ }^{14}$ These findings provide further evidence that uPA is more efficacious than tPA for fibrinolysis in IVH. Furthermore, an increasing number of clinical studies have proposed that uPA carries a high fibrinolytic potential without excitotoxicity, thus representing a novel, promising candidate 

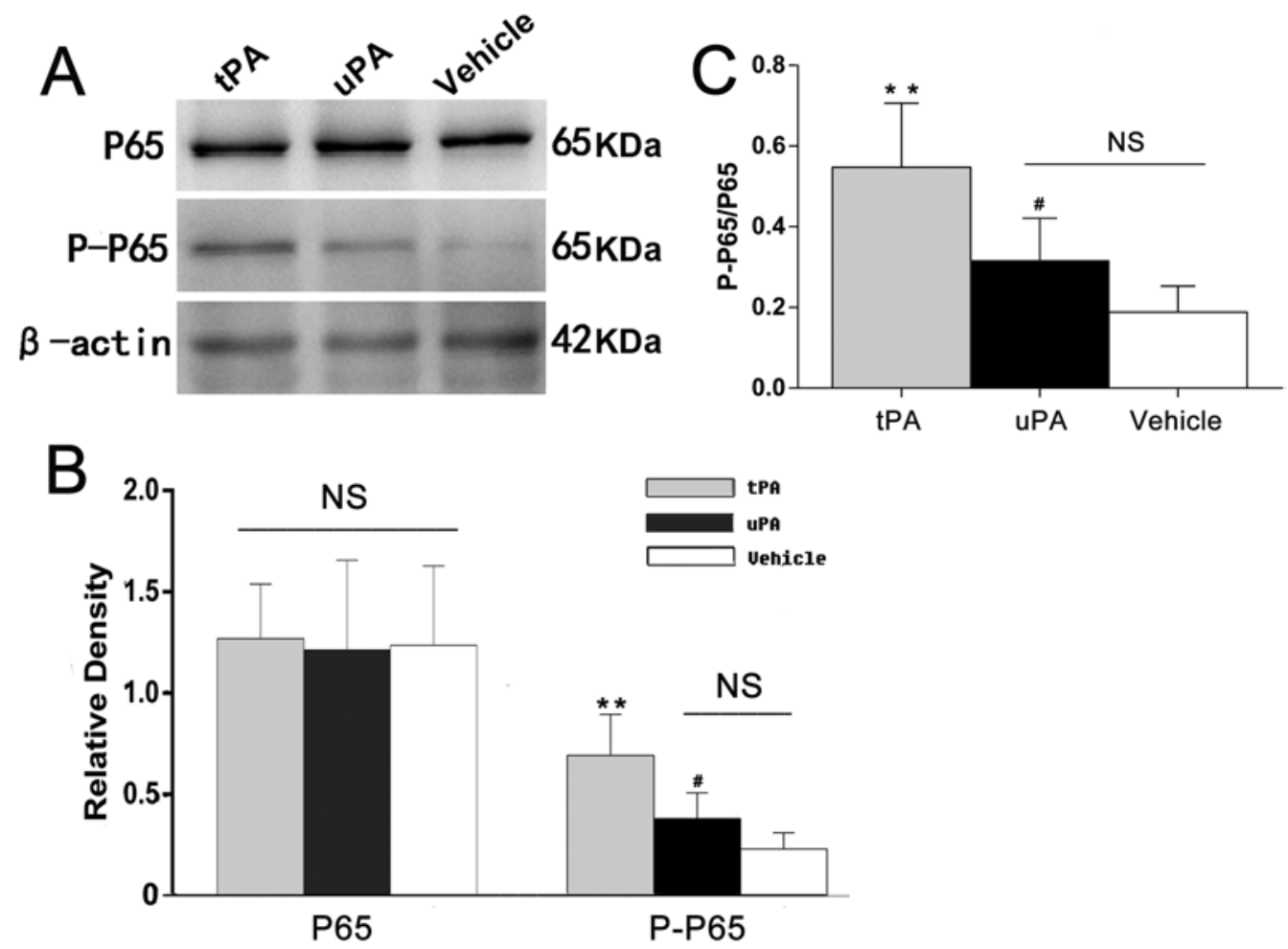

FIG. 5. Effect of tPA and UPA on NF-kB pathway activation after ICH. Representative Western blot analysis (A) and quantification of the relative density (B) of the band of total p65 and phospho-p65 (p-p65) in the perihematomal area on Day 3 after ICH. The $\mathrm{p}$-p65 protein expression levels $(\mathbf{C})$ were normalized to the p65 protein levels and quantification of the relative densities of each band (5 rats/group). ${ }^{*} p<0.05,{ }^{* *} p<0.01$ vs the vehicle group, $\# p<0.05$ vs the tPA group.

for fibrinolytic therapy for ICH. ${ }^{5,8,23,38}$ In agreement with the above publications, our results also revealed the greater efficacy of uPA, compared with tPA, in ameliorating PHE and motor function after ICH.

Claudin-5 and ZO-1 are important components of the BBB that maintain the normal function of the central nervous system. Matrix metalloproteinases are involved in the degradation of these proteins, thereby resulting in BBB disruption after $\mathrm{ICH} .{ }^{2}$ In our study, we observed the elevated expression of claudin-5 and ZO-1 (Fig. 4) following treatment with uPA and tPA in comparison with vehicle, which may be partly explained as more effectively downregulating MMP (MMP-2 and MMP-12; Fig. 3) expression in the 2 fibrinolytics groups. The EB extravasation test also demonstrated that the fibrinolytic groups protected BBB integrity and that the UPA was more effective (Fig. 2A and B). Meanwhile, we found that tPA increased MMP-9 expression more than uPA and vehicle did. Note that MMP-9 has been implicated in $\mathrm{ICH}^{1}$ and that tPA has been shown to activate MMPs through different mechanisms in which the NF- $\kappa \mathrm{B}$ pathway has a central role.,19 Therefore, we hypothesized that tPA and uPA could result in the differential expression of MMP-9 via the differential effects on the NF- $\mathrm{KB}$ pathway, thereby causing a different extent of BBB disruption. Then, we evaluated this hypothesis by Western blot analysis for NF- $\kappa \mathrm{B}$ activation and found that TPA showed a more remarkable activation of the NF- $\kappa$ B pathway than did uPA (Fig. 5) for ICH fibrinolysis, which may partly explain the reduced MMP-9 expression and improved BBB integrity in the uPA group.
In summary, both tPA and uPA protected the BBB by downregulating the MMPs, in which uPA was shown to be more efficacious by reducing activation of the NF- $\mathrm{KB}$ pathway. Because of limited evidence, more studies are needed to further validate the efficacy and safety of uPA for ICH fibrinolysis in larger preclinical research studies and clinical trials, particularly its comparison with tPA.

\section{Conclusions}

In the present study, we compared the use of 2 fibrinolytics, namely uPA versus tPA, in a rat ICH model. Our results showed that, despite showing a similar effect on hematoma reduction, uPA treatment better ameliorated PHE and improved the outcome following ICH than did tPA. Thus, uPA may be a promising candidate for fibrinolytic therapy for ICH.

\section{Acknowledgments}

We thank Dr. Fu Peishu from the pharmaceutical department of Southwest Hospital, Chongqing, for her diligent guidance in using the uPA and tPA during the whole study process. We are also grateful for the support from the Collaboration Innovation Center for Basic Science of China. This work was supported by Grant Nos. 81571130 (Z.C.), 81271281 (Z.C.), and 81371440 (Yang Yunfeng) from the National Natural Science Foundation of China and Grant No. 2014CB541606 (H.F.) from the National Key Basic Research Development Program (973 Program) of China.

\section{Reference}

1. Abilleira S, Montaner J, Molina CA, Monasterio J, Castillo J, 
Alvarez-Sabín J: Matrix metalloproteinase-9 concentration after spontaneous intracerebral hemorrhage. J Neurosurg 99:65-70, 2003

2. Ballabh P, Braun A, Nedergaard M: The blood-brain barrier: an overview: structure, regulation, and clinical implications. Neurobiol Dis 16:1-13, 2004

3. Candelario-Jalil E, Yang Y, Rosenberg GA: Diverse roles of matrix metalloproteinases and tissue inhibitors of metalloproteinases in neuroinflammation and cerebral ischemia. Neuroscience 158:983-994, 2009

4. Carhuapoma JR, Barrett RJ, Keyl PM, Hanley DF, Johnson RR: Stereotactic aspiration-thrombolysis of intracerebral hemorrhage and its impact on perihematoma brain edema. Neurocrit Care 8:322-329, 2008

5. Chang YH, Hwang SK: Frameless stereotactic aspiration for spontaneous intracerebral hemorrhage and subsequent fibrinolysis using urokinase. J Cerebrovasc Endovasc Neurosurg 16:5-10, 2014

6. Chen Q, Tang J, Tan L, Guo J, Tao Y, Li L, et al: Intracerebral hematoma contributes to hydrocephalus after intraventricular hemorrhage via aggravating iron accumulation. Stroke 46:2902-2908, 2015

7. Chen Q, Zhang J, Guo J, Tang J, Tao Y, Li L, et al: Chronic hydrocephalus and perihematomal tissue injury developed in a rat model of intracerebral hemorrhage with ventricular extension. Transl Stroke Res 6:125-132, 2015

8. Chen X, Chen W, Ma A, Wu X, Zheng J, Yu X, et al: Frameless stereotactic aspiration and subsequent fibrinolytic therapy for the treatment of spontaneous intracerebral haemorrhage. Br J Neurosurg 25:369-375, 2011

9. Chen Y, Zhang Y, Tang J, Liu F, Hu Q, Luo C, et al: Norrin protected blood-brain barrier via frizzled-4/ $\beta$-catenin pathway after subarachnoid hemorrhage in rats. Stroke 46:529536,2015

10. Chen Z, Gao C, Hua Y, Keep RF, Muraszko K, Xi G: Role of iron in brain injury after intraventricular hemorrhage. Stroke 42:465-470, 2011

11. Chen Z, Zhang J, Chen Q, Guo J, Zhu G, Feng H: Neuroprotective effects of edaravone after intraventricular hemorrhage in rats. Neuroreport 25:635-640, 2014

12. Gaberel T, Macrez R, Gauberti M, Montagne A, Hebert $\mathrm{M}$, Petersen KU, et al: Immunotherapy blocking the tissue plasminogen activator-dependent activation of N-methyl-Daspartate glutamate receptors improves hemorrhagic stroke outcome. Neuropharmacology 67:267-271, 2013

13. Gaberel T, Magheru C, Parienti JJ, Huttner HB, Vivien D, Emery E: Intraventricular fibrinolysis versus external ventricular drainage alone in intraventricular hemorrhage: a meta-analysis. Stroke 42:2776-2781, 2011

14. Gaberel T, Montagne A, Lesept F, Gauberti M, Lemarchand E, Orset C, et al: Urokinase versus Alteplase for intraventricular hemorrhage fibrinolysis. Neuropharmacology 85:158-165, 2014

15. Ghosh S, Karin M: Missing pieces in the NF-kappaB puzzle. Cell 109 Suppl:S81-S96, 2002

16. Guo J, Chen Q, Tang J, Zhang J, Tao Y, Li L, et al: Minocycline-induced attenuation of iron overload and brain injury after experimental germinal matrix hemorrhage. Brain Res 1594:115-124, 2015

17. Hayden MS, Ghosh S: Shared principles in NF-kappaB signaling. Cell 132:344-362, 2008

18. Hua Y, Schallert T, Keep RF, Wu J, Hoff JT, Xi G: Behavioral tests after intracerebral hemorrhage in the rat. Stroke 33:2478-2484, 2002

19. Jin R, Yang G, Li G: Molecular insights and therapeutic targets for blood-brain barrier disruption in ischemic stroke: critical role of matrix metalloproteinases and tissue-type plasminogen activator. Neurobiol Dis 38:376-385, 2010

20. Keep RF, Hua Y, Xi G: Intracerebral haemorrhage: mecha- nisms of injury and therapeutic targets. Lancet Neurol 11:720-731, 2012

21. Keric N, Maier GS, Samadani U, Kallenberg K, Dechent $\mathrm{P}$, Brueck W, et al: Tissue plasminogen activator induced delayed edema in experimental porcine intracranial hemorrhage: reduction with plasminogen activator inhibitor-1 administration. Transl Stroke Res 3 (Suppl 1):88-93, 2012

22. Li L, Tao Y, Tang J, Chen Q, Yang Y, Feng Z, et al: A cannabinoid receptor 2 agonist prevents thrombin-induced bloodbrain barrier damage via the inhibition of microglial activation and matrix metalloproteinase expression in rats. Transl Stroke Res 6:467-477, 2015

23. Lian L, Xu F, Hu Q, Liang Q, Zhu W, Kang H, et al: No exacerbation of perihematomal edema with intraclot urokinase in patients with spontaneous intracerebral hemorrhage. Acta Neurochir (Wien) 156:1735-1744, 2014

24. Miller CM, Vespa PM, McArthur DL, Hirt D, Etchepare M: Frameless stereotactic aspiration and thrombolysis of deep intracerebral hemorrhage is associated with reduced levels of extracellular cerebral glutamate and unchanged lactate pyruvate ratios. Neurocrit Care 6:22-29, 2007

25. Morgan T, Zuccarello M, Narayan R, Keyl P, Lane K, Hanley $\mathrm{D}$ : Preliminary findings of the minimally-invasive surgery plus rtPA for intracerebral hemorrhage evacuation (MISTIE) clinical trial. Acta Neurochir Suppl (Wien) 105:147-151, 2008

26. Mould WA, Carhuapoma JR, Muschelli J, Lane K, Morgan TC, McBee NA, et al: Minimally invasive surgery plus recombinant tissue-type plasminogen activator for intracerebral hemorrhage evacuation decreases perihematomal edema. Stroke 44:627-634, 2013

27. Narayan RK, Narayan TM, Katz DA, Kornblith PL, Murano G: Lysis of intracranial hematomas with urokinase in a rabbit model. J Neurosurg 62:580-586, 1985

28. Orakcioglu B, Becker K, Sakowitz OW, Herweh C, Köhrmann M, Huttner HB, et al: MRI of the perihemorrhagic zone in a rat $\mathrm{ICH}$ model: effect of hematoma evacuation. Neurocrit Care 8:448-455, 2008

29. Rohde V, Rohde I, Thiex R, Ince A, Jung A, Dückers G, et al: Fibrinolysis therapy achieved with tissue plasminogen activator and aspiration of the liquefied clot after experimental intracerebral hemorrhage: rapid reduction in hematoma volume but intensification of delayed edema formation. J Neurosurg 97:954-962, 2002

30. Tang J, Chen Q, Guo J, Yang L, Tao Y, Li L, et al: Minocycline attenuates neonatal germinal-matrix-hemorrhageinduced neuroinflammation and brain edema by activating cannabinoid receptor 2. Mol Neurobiol 53:1935-1948, 2016

31. Tang J, Tao Y, Tan L, Yang L, Niu Y, Chen Q, et al: Cannabinoid receptor 2 attenuates microglial accumulation and brain injury following germinal matrix hemorrhage via ERK dephosphorylation in vivo and in vitro. Neuropharmacology 95:424-433, 2015

32. Thai QA, Pradilla G, Legnani FG, Kretzer RM, Hsu W, Tamargo RJ: Lysis of intracerebral hematoma with stereotactically implanted tissue plasminogen activator polymers in a rabbit model. J Neurosurg 105:424-429, 2006

33. Thiex R, Küker W, Jungbluth P, Kayser C, Müller HD, Rohde I, et al: Minor inflammation after surgical evacuation compared with fibrinolytic therapy of experimental intracerebral hemorrhages. Neurol Res 27:493-498, 2005

34. Thiex R, Mayfrank L, Rohde V, Gilsbach JM, Tsirka SA: The role of endogenous versus exogenous tPA on edema formation in murine ICH. Exp Neurol 189:25-32, 2004

35. Thiex R, Weis J, Krings T, Barreiro S, Yakisikli-Alemi F, Gilsbach JM, et al: Addition of intravenous N-methyl-Daspartate receptor antagonists to local fibrinolytic therapy for the optimal treatment of experimental intracerebral hemorrhages. J Neurosurg 106:314-320, 2007 
36. van Asch CJ, Luitse MJ, Rinkel GJ, van der Tweel I, Algra A, Klijn CJ: Incidence, case fatality, and functional outcome of intracerebral haemorrhage over time, according to age, sex, and ethnic origin: a systematic review and meta-analysis. Lancet Neurol 9:167-176, 2010

37. Wagner KR, Xi G, Hua Y, Zuccarello M, de Courten-Myers GM, Broderick JP, et al: Ultra-early clot aspiration after lysis with tissue plasminogen activator in a porcine model of intracerebral hemorrhage: edema reduction and blood-brain barrier protection. J Neurosurg 90:491-498, 1999

38. Wang GQ, Li SQ, Huang YH, Zhang WW, Ruan WW, Qin $\mathrm{JZ}$, et al: Can minimally invasive puncture and drainage for hypertensive spontaneous Basal Ganglia intracerebral hemorrhage improve patient outcome: a prospective non-randomized comparative study. Mil Med Res 1:10, 2014

39. Xi G, Strahle J, Hua Y, Keep RF: Progress in translational research on intracerebral hemorrhage: is there an end in sight? Prog Neurobiol 115:45-63, 2014

40. Zhao H: Ischemic postconditioning as a novel avenue to protect against brain injury after stroke. J Cereb Blood Flow Metab 29:873-885, 2009

41. Zhao X, Sun G, Zhang J, Strong R, Song W, Gonzales N, et al: Hematoma resolution as a target for intracerebral hemorrhage treatment: role for peroxisome proliferator-activated receptor gamma in microglia/macrophages. Ann Neurol 61:352-362, 2007

42. Zhou H, Zhang Y, Liu L, Huang Y, Tang Y, Su J, et al: Mini- mally invasive stereotactic puncture and thrombolysis therapy improves long-term outcome after acute intracerebral hemorrhage. J Neurol 258:661-669, 2011

\section{Disclosures}

The authors report no conflict of interest concerning the materials or methods used in this study or the findings specified in this paper.

\section{Author Contributions}

Conception and design: Z Chen, Q Chen. Analysis and interpretation of data: Tan, Q Chen, Tao, Tang. Drafting the article: Tan, Q Chen. Critically revising the article: $\mathrm{Z}$ Chen, Tan, Niu, Tao, Yang, Guo, Zhu. Reviewed submitted version of manuscript: Z Chen, Li, H Feng, Zhu. Approved the final version of the manuscript on behalf of all authors: Z Chen. Statistical analysis: Z Feng, Li, Tang. Administrative/technical/material support: Tan, Niu, Z Feng, Yang, Guo, H Feng. Study supervision: Z Chen, H Feng.

\section{Correspondence}

Zhi Chen, Department of Neurosurgery, Southwest Hospital, Third Military Medical University, No. 30, Gaotanyan St., Chongqing, 400038, The People's Republic of China. email: zhichen@tmmu.edu.cn. 